\section{Note on Regulatory Toxicology Requirements for Adjuvants and Vaccines; in View of the Newly Established WHO Guidelines}

Received: Oct 09, 2015, Accepted: Oct 31, 2015, Published: Nov 07, 2015

\section{Overview and Background of the 2014 WHO Guidelines}

While the use of vaccines for the treatment of noninfectious disease is being recognized, the current guidelines on nonclinical safety assessment of vaccines mainly focus on vaccines designed for prevention of infectious diseases. Guidelines on nonclinical safety assessment of preventive vaccines for infectious disease were first published by the EMA [1], followed by the WHO [2] and then FDA [3] (only for reproductive and developmental toxicity studies). In Japan, the Ministry of Health, Labour and Welfare (MHLW) created the guidelines on the nonclinical evaluation of vaccines for infectious disease (hereafter, the Japanese Guidelines) [4, 5], along with the issuance of clinical guidelines.

Comparing the present vaccination scenario with that approximately a decade ago when these guidelines were initially issued, a major difference is the use of vaccine adjuvants. Adjuvants increase the effectiveness of vaccines by potentiating the immune response, and their use in vaccines has significantly increased. In recent years, in addition to traditional aluminumcontaining adjuvants, diverse types of adjuvants such as tolllike receptor (TLR) agonist, oil-in-water emulsions, nanocarriers (particulate, liposomes, polymeric etc.) [6] and others [7] have become available (hereafter, novel adjuvants). For instance, the number of vaccines approved in Japan from 1985 to 2005 was eight, and two of them (25\%) contained adjuvants, and both of them were aluminum-based. In contrast, that number from 2006 to 2015 was twenty-five, and twelve of them (48\%) contained adjuvants, where four (33\%) of them were novel adjuvants. Although the EMA has issued guidelines specific to adjuvants in vaccines [8], concerns were raised that it did not completely addressed the current situation. In Japan, interests have been raised for establishing the specific position of adjuvants, because they are still regarded as additives in its regulations [9]. Under these situations, the WHO guidelines on the nonclinical evaluation of vaccine adjuvants and adjuvanted vaccines were established on November 21, 2014 (hereafter, the 2014 WHO Guidelines) [10].

One unique feature of the WHO guidelines is that 'the authors' are listed at the end of a document, also with every drafting step. For example, the number of the representative participants of the WHO consultation held in headquarter of WHO in Geneva,
Mineo Matsumoto ${ }^{1}$ Shinichi Komatsu², Hajime Matsui ${ }^{3}$, Kazuhiro Shimomura ${ }^{4}$, Kazuto Watanabe ${ }^{5}$, Kohei Amakasu', Osamu Fueki', Jun-ichi Sawada', Kazushige Maki', Kazutoshi Shinoda' ${ }^{1}$ and Hiroshi Onodera' ${ }^{1}$

1 Review Division, Pharmaceuticals and Medical Devices Agency (PMDA) $^{*}$, Kasumigaseki 3-3-2, Chiyoda-ku, Tokyo 100-0013, Japan

2 Research and Development Department, POC Clinical Research Inc. Taishidou 4-11, Setagaya-ku, Tokyo 154-0004, Japan

3 Pathology Department, The ChemoSero-Therapeutic Research Institute, Kyokushi Kawabe 1314-1, Kikuchi-shi, Kumamoto 869-1298, Japan

4 Vaccine Research Laboratories, Kitasato Daiichi Sankyo Vaccine Co. Ltd., KitaKasai 1-16-13, Edogawa-ku, Tokyo 1348630, Japan

5 Research Division, Chugai Pharmaceutical Co. Ltd., Komakado, 1-135, Gotemba, Shizuoka 412-8513, Japan

The views expressed in this article are those of the authors and do not necessarily reflect the official views of Pharmaceuticals and Medical Devices Agency.

Corresponding author: Mineo Matsumoto

Review Division, Pharmaceuticals \& Medical Devices Agency (PMDA) ${ }^{\text {th }}$, Kasumigaseki 3-3-2, Chiyoda-ku, Tokyo 100-0013, Japan

= matsumoto-mineo@pmda.go.jp

Tel: +81335069555

Fax: +8133506 9467 
with the aim of finalizing the WHO guidelines on nonclinical evaluation of vaccines published in 2005 (hereafter, the 2005 WHO Guidelines) [2] is 12 (regulatory body), 9 (academia) and 7 (industry) from 17 countries, whereas that of the 2014 WHO Guidelines is 18,5 and 15 from 23 countries, respectively. This indicates that a wider range of contributions was made to create the 2014 WHO Guidelines, especially from industry side (25\% in 2005 vs. $39 \%$ in 2014).

The 2014 WHO Guidelines are based on a careful consideration of the other abovementioned guidelines $[1,4,8]$. Furthermore, when compared with the 2005 WHO Guidelines [2], the following sections were newly added in the 2014 WHO Guidelines: "Rationale for the use of the adjuvant", "Toxicity studies of adjuvant alone", "Autoimmune disease induced by adjuvants" and "Considerations for the first-in-human clinical trials". With respect to the topic of "Autoimmune disease induced by adjuvants," discussions by the International Life Science Institute/Health and Environmental Sciences Institute (ILSI/HESI) on the adjuvants and autoimmune project, which occurred almost concurrently with the establishment activities on the 2014 WHO Guidelines, were particularly important (see below). The provisions of the 2014 WHO Guidelines regarding nonclinical evaluation of adjuvants and vaccines consider the $3 R$ s (replacement, reduction, and refinement) principle for animal research. The points of issues raised during the establishment of the 2014 WHO Guidelines, particularly differences compared to the 2005 WHO Guidelines, will be discussed below (Table 1). This article reflects the results of the research group for the Japan Agency for Medical Research and Development (AMED)-sponsored research on international harmonization of regulations for quality, efficacy, and safety of pharmaceutical products (Akiyoshi Nishikawa, Director, Biological Safety Research Center, National Institute of Health Sciences) and our working subgroup for the development of nonclinical vaccine evaluation guidelines (hereafter, AMED-Nonclinical Vaccine Evaluation Group).

\section{The Points of Issues Raised During the Establishment of the 2014 WHO Guidelines}

\section{Scope of the guidelines}

The scope of the Guidelines is described such that it covers adjuvanted vaccines for both prophylactic and therapeutic uses against infectious diseases. This definition does not differ from the 2005 WHO Guidelines and consistent with the Japanese Guidelines. It is assumed that adjuvanted vaccines for the therapeutic use against infectious diseases are referring to the treatment against viruses causing hepatitis B, varicella-zoster, and rabies.

However, the 2014 WHO Guidelines added the description
Citation: Matsumoto $\mathrm{M}$, Komatsu S, Matsui $\mathrm{H}$, et al. Note on Regulatory Toxicology Requirements for Adjuvants and Vaccines; in View of the Newly Established WHO Guidelines. J Med toxicol clin forensic med. 2016, 1:2.

that some of the outlined principles may be applicable to the adjuvanted therapeutic vaccines for non-infectious diseases as well (e.g., cancer). In other words, the scope of the 2014 WHO Guidelines has slightly expanded in comparison to the previous version [2]. Given this widened scope, the AMED-Nonclinical Vaccine Evaluation Group assumes that the "local reactogenicity evaluation" in the 2014 WHO Guidelines can be applied to therapeutic vaccines for noninfectious diseases. On the other hand, the group expresses the opinion that "selection of animal species" and "toxicity studies of adjuvant alone" would not be applicable as is for therapeutic vaccines for non-infectious diseases. That says, in therapeutic vaccines for non-infectious diseases, it is not feasible to replicate immune responses in animals due to the strong dependence on HLA. Thus, the required non-clinical safety studies are characterized as those detecting off-target toxicity rather than on-target toxicity [11].

\section{Dosing frequency in repeated-dose toxicity studies of adjuvanted vaccines}

In comparing previous guidelines $[1,4,8]$ for the number of doses in repeated-dose toxicity studies, there have been two divergent guidelines: (1) a concept that a dosing frequency equal to the number of doses proposed in clinical usage is acceptable and should "be over" the proposed frequency and (2) a concept that dosing frequency equal to the number of doses proposed for clinical usage is not acceptable and should exceed the number of doses proposed for clinical usage by at least one dose (socalled "N+1 rule"; $\mathrm{N}=$ number of doses proposed in clinical usage). Up to now, as opposed to concept (1) adapted from the 2005 WHO Guidelines [2], the Japanese Guidelines [4] has adopted concept (2) in which dosing frequency should exceed the planned number for the clinical trial. In comparison, the EMA Guidelines [1] have adopted concept (1), while the FDA Guidelines [3] have no guidance on the matter of dosing frequency. However, both regulatory bodies have declared the support for concept (2) in the published paper [5]. The basis for support for "N+1 rule" in concept (2) is that toxicity studies must investigate toxicity profiles under stricter conditions than expected in a clinical setting. Furthermore, as a dosing frequency may be increased during the vaccine development phase and post-approval, the repeated-dose toxicity studies should include the additional dose to prepare for the anticipated increase in dosing frequency.

The 2014 WHO Guidelines took into consideration opinions from the above regulatory agencies and incorporate concept (2) to concept (1) in the guidelines. That says like, "The number of doses in repeated-dose toxicity studies should equal or exceed that for humans. However, the studies are often designed to include one dose more than planned for the clinical trial to allow for the possible inclusion of an additional dose in the clinicals" [10]. 
Table 1 Differences in specific issues between the 2005- and 2014 WHO Guidelines.

\begin{tabular}{|c|c|c|c|}
\hline & Items in the guidelines & 2005 WHO Guidelines & 2014 WHO Guidelines \\
\hline 1 & scope of the guidelines & $\begin{array}{l}\text { (no indication about the applicability to } \\
\text { noninfectious diseases) }\end{array}$ & $\begin{array}{l}\text { principles may be applicable to therapeutic vaccines } \\
\text { against noninfectious diseases (e.g., cancer) }\end{array}$ \\
\hline 2 & dosing freq in repeat-dose tox & $\begin{array}{c}\text { number of doses should be equal to or more } \\
\text { than that in humans }\end{array}$ & 'N+1 rule' is suggested \\
\hline 3 & dosing period in DART & pretreatment is frequently required & $\begin{array}{c}\text { dose on day } 1 \text { of pregnancy is preferable to pre } \\
\text { treatment }\end{array}$ \\
\hline 4 & tox study of novel adjuvant alone & required (w/ 2 animal species) & $\begin{array}{l}\text { can be included in tox study of vaccine formula } \\
\qquad(w / 1 \text { animal species) }\end{array}$ \\
\hline 5 & autoimmune disease by adjuvant & (no indication) & no clear recommendation \\
\hline
\end{tabular}

\section{Dosing period for adjuvanted vaccines in reproductive and developmental toxicity studies}

In contrast to the typical three-study design (Fertility, Pre/ postnatal, Embryo/fetal Study) that is adequate for reproductive and developmental toxicity (DART) studies of low molecular medicines, the combined dosing period of Fertility and Pre/ postnatal studies is often used for the DART studies involving vaccines. The basis for this approach is that in contrast to low molecular compounds, regardless the addition of adjuvants, vaccines as a cause of DART is not generally a major concern. Even FDA Guidelines, which have the most detailed requirements for vaccine DART studies, are based on this single study design [3].

A divergence of the 2014 WHO Guidelines from the previous guidelines was the dosing period for the single study design. Specifically, the requirement of dosing prior to mating was revised. In the 2005 WHO Guidelines, it was stated as premating treatment is most likely required [2]; this requirement was also included in the FDA Guidelines [3]. However, the 2014 WHO Guidelines revised the content to in the view of the effect of adjuvant on early pregnancy parameters, rather than dosing prior to mating, it is recommended to dose animals on day 1 of pregnancy (confirmation date of copulation: day 0 of gestation). With respect to the basis for the above change, the primary factor may be seen in the explanation as; some adjuvants may have concern about an adjuvant-induced systemic inflammatory response (e.g., fever) that could adversely affect early pregnancy. That is, with respect to reproductive and developmental toxicity associated with adjuvanted vaccines, the prevailing two theories for cause of toxicity are (1) antibody induced by active ingredients of vaccine (vaccine-induced antibody) and (2) adjuvant that is added to vaccine. Based on the abovementioned description, currently, it is conjectured that toxicity associated with (2) adjuvant is tending to be more focused rather than (1) vaccineinduced antibody.

\section{Toxicity studies of novel adjuvant alone}

An important consideration in the evaluation of a novel adjuvant for vaccines is how to assess the adjuvant toxicity. The $2005 \mathrm{WHO}$ Guidelines stipulated such that if no toxicological data is present for a new adjuvant, toxicity study of the adjuvant alone should be performed primarily. However, in the 2014 WHO Guidelines, it has been modified to state as although evaluation of the adjuvant alone can be significant for novel adjuvants, a study arm receiving adjuvant alone may also be included in the toxicity studies of vaccine formula. With respect to the safety of new adjuvants, this should be associated with the growing consensus of the importance of understanding the potential toxicity of adjuvants just as a part of the toxicities of new vaccine formulations, as well as the $3 R$ s principle.

Next, for evaluating the toxicity of a new adjuvant, we need to consider the number of animal species to be used for general toxicity and DART studies. On this matter, the 2005 WHO Guidelines [2] and the EMA guidelines for adjuvants [8] recommended one rodent species and one non-rodent animal species, i.e., a total of two animal species, which are the same as those for toxicity studies of low molecular medicine. In contrast, the 2014 WHO Guidelines did not emphasize this requirement; however, based on the abovementioned explanation as "a study arm receiving adjuvant alone may also be included in the toxicity studies of vaccine formula" and "the use of an relevant animal species, i.e., an animal species that is responsive to the vaccine is recommended for the toxicity evaluation of adjuvanted vaccine" [10], it can be interpreted that using single species is basically acceptable even for a toxicity assessment of a new adjuvant alone. This requirement for number of animal species is considered virtually equivalent to the case with vaccine formulation. These differences in toxicity assessment of a new adjuvant alone are one of the most significant changes from the 2005 WHO Guidelines.

\section{Autoimmune disease induced by adjuvant}

The practical action of adjuvants to enhance the immune response to antigen is presumed to result in a safety concern that autoimmune disease may be triggered. For example, "Gulf War Syndrome" developed in the US troops was reported to be associated with the administration of adjuvant for anthrax vaccination [12]. On the matter of adjuvant safety, the ILSI/ HESI discussed "Adjuvant and autoimmune disease" (workshop was held under same title in Amsterdam, the Netherlands on October 18-19, 2014) along with the work on the establishment of the 2014 WHO Guidelines. The outcome of the ILSI/HESI workshop was reflected in the 2014 WHO Guidelines. A study reported that when an adjuvant, pristane, an impurity found in natural oil extracted from shark liver, was single administered to mice, it was found to activate production of autoantibodies found in human systemic lupus erythematous patients [13]. The autoantibody production is considered to be dependent on the Type I-IFN and TLR 7. Furthermore, when the vaccine adjuvant, squalene, an unsaponifiable unsaturated hydrocarbon found in 
shark liver oil, or incomplete Freund's adjuvant was administered to mice, autoantibody production was enhanced; this suggested that this model is useful in understanding the development of autoimmune diseases probably occurring in humans after exposure to adjuvants [13]. However, the ILSI/HESI workshop concluded that based on significant differences in adjuvant composition, dosage level, administration route, and other factors from the vaccination applied in humans, extrapolation of the results from these models to humans should be conducted cautiously at present [14]

Based on the preceding discussion, the following explanations were added to the 2014 WHO Guidelines: no compelling clinical evidence are found that adjuvants could lead to the induction of autoimmune disease, and established animal models for this matter do not currently exist. Therefore, the guidelines reached the conclusion that no recommendations can be made at present regarding specific nonclinical studies, and that these are complex and multifactorial conditions; further research is required to identify additional biomarkers [10].

\section{Conclusion}

In recent years, vaccine antigens have been combined with novel adjuvants to enhance the immune response induced by the vaccine antigen. The nonclinical safety assessment of adjuvants and/or adjuvanted vaccines would be challenged by the species specific actions of adjuvants together with their particular physicochemical characteristics apart from the vaccine active ingredients. The release of the 2014 WHO Guidelines was timely in light of this situation. The primary differences between the 2005 and the 2014 WHO Guidelines are represented by 'dosing frequency in repeated-dose toxicity studies of adjuvanted vaccines', 'dosing period for adjuvanted vaccines in reproductive and developmental toxicity studies' and 'toxicity studies of new adjuvant alone', etc. These indications are expected to adhere to unsolved problems raised in the paper [5] especially with regard to the use of novel adjuvants. The recommendations newly introduced into the 2014 WHO Guidelines are also expected to have minimized differences in the regulation of nonclinical safety issues of preventive vaccines among three $\mathrm{ICH}$ regions [5].

\section{Acknowledgment}

The authors wish to acknowledge the support of Drs. Ken Ishii (Osaka University), Tsutomu Urano, Naoyuki Yasuda and Takeyuki Satoh (PMDA) for this work. 


\section{References}

1 European Medicines Agency (EMA) (1998) Note for guidance on preclinical pharmacological and toxicological testing of vaccines. Committee for Proprietary Medicinal Products.

2 World Health Organization (WHO) (2005) Guidelines on nonclinical evaluation of vaccines. WHO Technical Report Series.

3 Food and Drug Administration (FDA) (2006) Guidance for Industry: Considerations for Developmental Toxicity Studies for Preventive and Therapeutic Vaccines for Infectious Disease Indications.

4 (2012) Guidelines for Nonclinical Studies of Preventive Vaccines for Infectious Diseases. Notification of yakushokushinsahatsu.

5 Sun Y, Gruber M, Matsumoto M (2012) Overview of global regulatory requirements for vaccines and adjuvants. J Pharm Tox 65: 49-57.

6 Kuroda E, Coban C, Ishii KJ (2013) Particulate adjuvant and innate immunity: past achievements, present findings, and future prospects. International Rev Immunol 32: 209-220.

7 Silva FTD, Pasquale AD, Yarzabal JP, Garcon N (2015) Safety assessment of adjuvanted vaccines: Methodological considerations. Hum Vaccin Immunother 11: 1814-1824.
8 European Agency for the Evaluation of Medicine (EMEA) (2005) Guideline on adjuvants in vaccines for human use. EMEA.

9 Matsumoto M (2014) Considerations for specific position of adjuvants under Japanese pharmaceutical affairs. Reg Sci Med Products 3: 175.

10 World Health Organization (WHO) (2014) Guidelines on the nonclinical evaluation of vaccine adjuvants and adjuvanted vaccines.

11 Matsumoto $M$, Komatsu S, Tsuchimoto $M$, Matsui $H$, Watanabe $\mathrm{K}$, et al. Considerations for non-clinical safety studies of therapeutic peptide vaccines. Reg Tox Pharm 70: 254-260.

12 Matsumoto G (2004) Vaccine A: The covert government experiment that's killing our soldiers and why Gl's are the only first victims. BasicBooks.

13 Satoh M, Kuroda Y, Yoshida H, Behney KM, Mizutani A, et al. (2003) Induction of lupus autoantibodies by adjuvants. J Autoimmun 21: 1-9

14 van der Laan JW, Gould S, Tanir JY (2015) Safety of vaccine adjuvants: focus on autoimmunity. Vaccine 33: 1507-1514. 